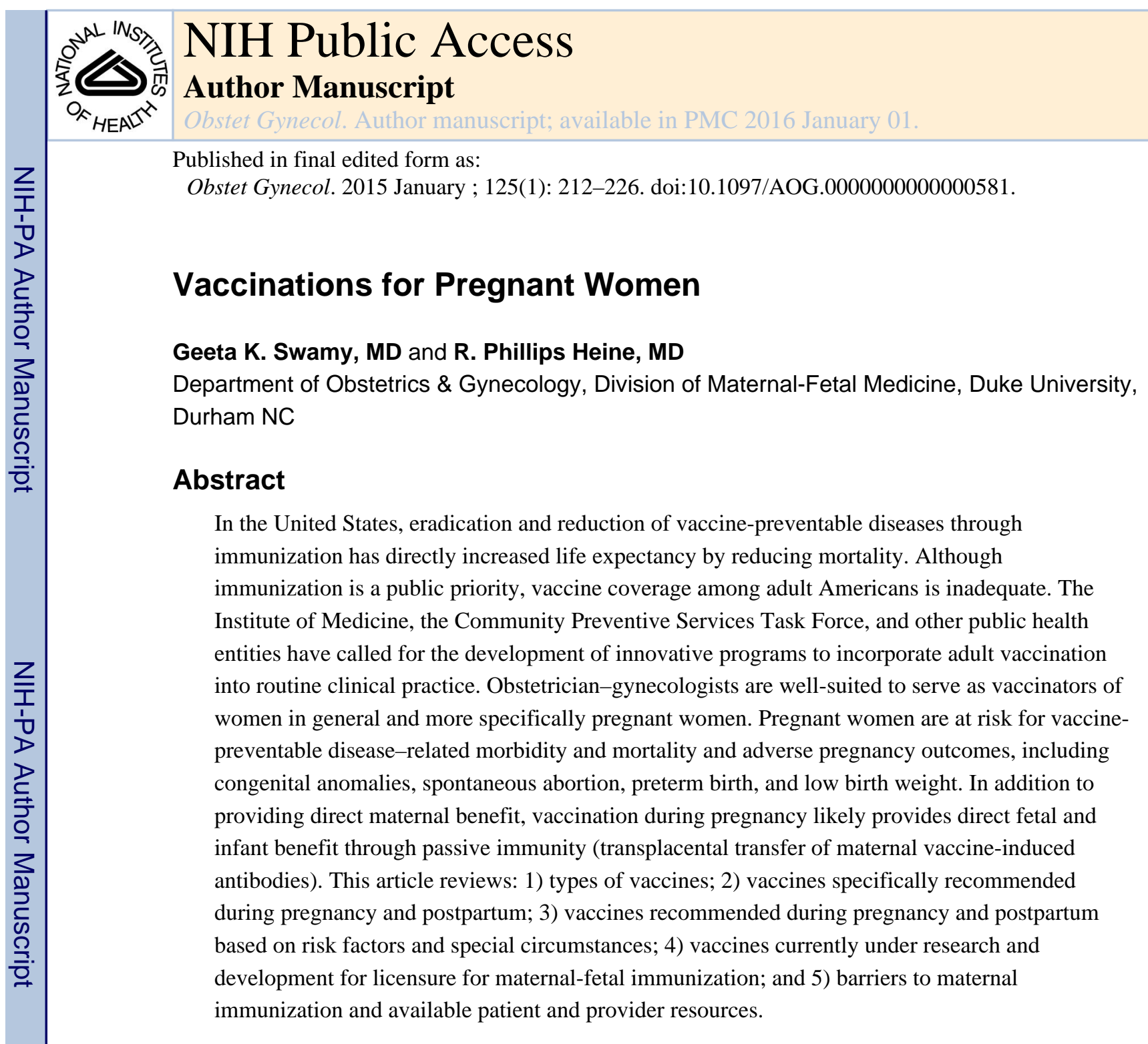

\title{
Introduction
}

\begin{abstract}
Vaccine-preventable diseases are defined as those infectious diseases for which an effective preventive vaccine exists and may be prevented by vaccinating individuals per standard recommendations. ${ }^{1}$ In the United States, eradication and reduction of vaccine-preventable diseases through immunization has directly increased life expectancy by reducing infectious disease mortality. ${ }^{2}$ For example, deaths from invasive pneumococcal disease, hepatitis A and B, and varicella have dramatically declined over the last decade. ${ }^{3}$ Infectious diseases remain a major cause of adult morbidity and mortality, with well over 50,000 adults dying each year from vaccine-preventable diseases and associated complications. ${ }^{3}$
\end{abstract}

Corresponding Author: Geeta K. Swamy, MD, Duke Maternal-Fetal Medicine, 2608 Erwin Rd, Suite 200, Durham, NC 27705, 919-668-1600 (O) 919-681-7861 (fax), geeta.swamy@ duke.edu.

Financial Disclosure: Geeta K. Swamy has received past support from GlaxoSmithKline for consultant and speaker activities that ended in March 2012. R. Phillips Heine has been a consultant and speaker for GlaxoSmithKline and a speaker for Merck, Inc. Both authors have received research funding from the National Institutes of Health, the Centers for Disease Control and Prevention, the American College of Obstetricians and Gynecologists, and GlaxoSmithKline to conduct vaccine-related research. 
National immunization recommendations currently target 17 vaccine-preventable diseases across the lifespan. However, adult coverage for most routinely recommended vaccines is suboptimal and well below Healthy People 2020 goals. ${ }^{4}$ In 2011, only $20 \%$ of adults were appropriately vaccinated against pneumococcus, $12.5 \%$ against tetanus, diphtheria, and pertussis or Hepatitis A, 35\% against hepatitis B, and $43 \%$ of women against human papillomavirus (HPV). ${ }^{5}$ Considerable increases in vaccination coverage are needed to significantly reduce or eradicate the incidence of vaccine-preventable diseases in adults. The Institute of Medicine, the Community Preventive Services Task Force, and other public health entities have called for the development of innovative programs and incorporation of adult vaccination into routine clinical practice. ${ }^{6,7}$ With approximately 32,000 obstetrics and gynecology offices in the United States, integration of adult immunizations into routine obstetric and gynecology practice is one approach that would reach the vast majority of women across the lifespan.

Obstetrician-gynecologists (ob-gyns) provide more general medical care to women than either family practice or internal medicine providers, and thus would have opportunities to incorporate vaccination into standard clinical care. ${ }^{8}$ Moreover, ob-gyns specifically care for pregnant women who, along with their fetuses, are particularly vulnerable to vaccinepreventable disease-related complications. Pregnant women are at extremely high risk for influenza-related morbidity and mortality including adverse pregnancy outcomes (fetal growth restriction, preterm birth, and fetal demise). ${ }^{9-13}$ Rubella and varicella infections during pregnancy can lead to complex congenital anomalies. Although congenital hepatitis B does not cause malformations, vertical transmission is associated with life-long disease and long-term sequelae. Thus, immunization prior to conception would be ideal for the prevention of vaccine-preventable diseases associated with congenital disease. However, for vaccine-preventable diseases associated with adverse maternal, fetal, or infant health, immunization during pregnancy provides not only maternal benefit but may have the added benefit of direct infant protection. Passive immunity - transplacental immune globulin (Ig)G antibody transfer from vaccinated mother to fetus - provides infant protection up to 6 months of life. ${ }^{14,15}$ Maternal immunization is particularly important when considering vaccine-preventable diseases for which we have no other options for protecting young infants, such as influenza and pertussis. Specifically, influenza vaccine is not licensed for use prior to 6 months of age, and adequate antipertussis antibodies are only achieved after 2-3 doses of diphtheria and tetanus toxoids and acelluar pertussis vaccine (DTaP) by 6 months of age. ${ }^{16}$

Ob-gyns are well-positioned to screen and vaccinate pregnant women against vaccinepreventable diseases, having demonstrated their capability through the near-eradication of congenital rubella in the United Staters through routine antenatal surveillance and postpartum vaccination. Further, ob-gyns were heavily involved in the extensive administration of H1N1 vaccine to pregnant women during the 2009 pandemic and have continued their efforts to increase maternal influenza vaccination, exceeding 50\% coverage for the first time in 2012-2013. ${ }^{17}$ Among the vaccines recommended by the Centers for Disease Control \& Prevention (CDC) for adults, two are directly recommended for administration during pregnancy, four are recommended in pregnancy based on additional risk factors, and two are specifically recommended during the postpartum period. ${ }^{18}$ With 
nearly 4 million U.S. births each year, ob-gyns have the potential to greatly affect the health and well-being of mothers and infants through maternal immunization and contribute to the public health campaign for adult vaccine coverage. This article describes: 1) types of vaccines; 2) vaccines specifically recommended during pregnancy and postpartum;3) vaccines recommended during pregnancy and postpartum under based on risk factors and special circumstances; 4) vaccines currently under research and development for licensure for maternal-fetal immunization; and 5) barriers to maternal immunization and available resources.

\section{Types of Vaccines}

Based on laboratory methods, vaccines are classified as live attenuated, inactivated or killed, toxoid, subunit or conjugate vaccines (Table 1). Live attenuated vaccines may contain living organisms that have been weakened or altered so as not to cause infection. ${ }^{19,20}$ Diseasecausing pathogens are manipulated in the laboratory through multiple rounds of tissue culture or animal embryos, mutagenesis, or targeted genetic alterations to specifically select very low virulent strains. Manipulated pathogens do not cause overt human disease, but adequately stimulate an immune response, so that healthy people mount an immune response nearly equivalent to what would follow natural exposure or infection. Though technically possible for clinical infection to occur after live attenuated vaccination, it is extremely uncommon, and any illness that occurs is typically much milder than natural infection and is classified as an adverse reaction. However, immunocompromised individuals are at risk for unregulated pathogen replication followed by severe to fatal infection and cannot receive live attenuated vaccines. Similarly, live attenuated vaccines are contraindicated during pregnancy due to the theoretical risk of perinatal infection resulting in congenital disease, eg, rubella or varicella.

Inactivated or killed vaccines are created by inactivating a pathogen using heat or chemicals like formalin or formaldehyde. ${ }^{19,20}$ Inactivating processes destroy the pathogen's ability to replicate, as it does not contain any living or infectious particles and thus cannot result in clinical infection. Certain bacteria cause disease by producing a toxin rather than by direct bacterial interaction, as seem in Clostridium tetani. Vaccines against such diseases, called toxoid vaccines, are similarly created by inactivating the toxins using heat or chemicals. Inactivated and toxoid vaccines maintain their ability to generate an immune response, albeit less robust than the response after live attenuated vaccines. Antibody levels wane over time, thus requiring multiple dosing, booster dosing, or both to maintain adequate protection.

Subunit vaccines contain fragments of the pathogens they protect against, which subsequently provoke a protective immune response. ${ }^{19,20}$ One method for creating a subunit vaccine is by isolating a specific antigenic protein as with acellular pertussis vaccine. Alternatively, several subunit vaccines are created through genetic engineering or recombination. For hepatitis B vaccine example, a gene encoding for a pathogen-specific protein is inserted into another virus or cell culture. For HPV vaccine, expression of a single HPV protein results in production of virus-like particles. Virus-like particles contain no actual viral genetic material and thus cannot cause infection. After vaccination, the immune system recognizes the expressed proteins from recombinant vaccines and develops 
protective antibodies against the target pathogen. Conjugate vaccines are similar to recombinant vaccines except they are created by chemically combining pieces of the bacterial coat with a carrier protein. The bacterial components are unable to cause infection and require the carrier protein to stimulate an adequate immune response.

\section{Vaccines Recommended for Use in Pregnancy and Postpartum (Table 1)}

\section{Influenza}

Influenza (flu), an RNA virus with A and B serotypes, and is responsible for both endemic and pandemic flu. Both types are responsible for endemic flu while type A, due to antigenic drift of its surface proteins hemagglutinin and neuraminidase, is responsible for flu pandemics. ${ }^{21}$ In a single flu season it is estimated that $20 \%$ of the U.S. population contracts the illness, with the number increasing to 50\% during pandemics. Each year, 20\% of pregnant women will develop upper respiratory-like illness, with $10 \%$ having laboratoryconfirmed flu. ${ }^{22}$

Pregnant women infected with flu have increased rates of hospitalization, cardiopulmonary complications, and death when compared to the general population. ${ }^{12}$ Complications are further increased during pandemics, as seen during the $2009 \mathrm{H} 1 \mathrm{~N} 1$ outbreak when $5 \%$ of all related deaths occurred in pregnant women, yet they only encompass $1 \%$ of the total population. ${ }^{11}$ Multiple complications including spontaneous abortion, stillbirth, neonatal death, preterm birth, and low birth weight have been reported among influenza-infected women and likely correlate with disease severity. ${ }^{13}$ During the 2009 pandemic, the preterm birth rate increased threefold among infected pregnant women admitted to the hospital. ${ }^{23}$

Immunization is the best strategy for flu prevention as supported by the current CDC recommendation that all pregnant women receive inactivated influenza vaccine (IIV) during flu season. Live attenuated vaccine is contraindicated in pregnancy, though inadvertent administration during the first trimester has not been associated with adverse outcomes. ${ }^{24}$ Regarding IIV efficacy in pregnancy, a recent randomized study of 340 pregnant women revealed a $36 \%$ reduction in influenza illness. ${ }^{14} \mathrm{~A}$ large population based study from Norway revealed a $70 \%$ reduction in flu illness in immunized mothers, suggesting an even greater maternal benefit than previously thought. ${ }^{10}$ In addition to maternal benefits, IIV during pregnancy carries significant fetal and neonatal benefits. Maternal immunization has been associated with an increase in birth weight and a reduction in low birth weight, preterm birth, and fetal death. ${ }^{10,14}$ Further, a recent randomized trial of third-trimester maternal IIV compared with pneumococcal vaccine led to a $29 \%$ reduction in respiratory illness and a $63 \%$ reduction in laboratory-confirmed flu among newborns up to 6 months of age in the maternal IIV group. ${ }^{14}$ These findings have been confirmed in multiple population-based studies, with confirmed flu cases reduced $39-47 \%$ and hospitalizations reduced up to $90 \%$ in infants up to 6 months of age. ${ }^{25,26}$ It is unknown whether maternal immunization during the first or second trimester will provide similar infant benefit. Future studies are required to address gestational age timing of vaccination as timing of administration may differ for maternal, fetal, or neonatal benefit. 
Occurring in more than $90 \%$ of infected individuals, the most common symptoms of flu are fever and cough, with sore throat, myalgia, and headache occurring less commonly in $~ 30$ $50 \%$ of patients. Pregnant women who present with fever above $100.0^{\circ} \mathrm{F}$, exhibit either sore throat or cough, and have no other explanatory causes can be presumptively diagnosed with the flu, and treatment should be initiated. Rapid tests are available, but poor sensitivity limits usefulness in high-risk populations such as pregnant women. ${ }^{27}$ Confirmatory tests such as culture or polymerase chain reaction are extremely sensitive for the diagnosis of the flu but the delay in result reporting and lack of widespread availability limit their utility. According to the CDC, treatment should not be delayed while waiting for a result. ${ }^{28}$ However, confirmatory testing remains important for public health surveillance of serotype and identification of drug resistance patterns.

Treatment of flu during pregnancy consists of oral (oseltamivir) or inhaled (zanamivir) therapy. Oseltamivir is preferred in pregnancy due to its safety profile and convenience unless high levels of osteltamivir resistance are encountered, then shifting preference to inhaled zanamivir. ${ }^{29}$ Treatment should be initiated as rapidly as possible, as delay in initiation (more than 2 days of symptom onset) has been associated with worsening morbidity (ie, ICU admission, mechanical intubation, and death).

\section{Tetanus, Diphtheria, and Pertussis}

Vaccination against diphtheria, tetanus, and pertussis is included in the standard U.S. childhood vaccination schedule with the diphtheria, tetanus, acellular pertussis (DTaP) series starting at 2 months of age. C. tetani releases the tetanospasmin neurotoxin, which causes tetanus infection or prolonged muscular contraction. Corynebacterium diphtheriae causes an upper respiratory infection called diphtheria. Tetanus and diphtheria infections are nearly eradicated in the United States through routine decennial Td booster dosing across the lifespan and exposure-related administration. However, pertussis - a respiratory infection caused by Bordetella pertussis - has not followed a similar pattern. Since the 1980s, pertussis has been on the rise in the United States after many years of effective disease control and prevention. From less than 5,000 reported cases in the early 1980s, pertussis reached to an all-time high of 48,277 cases in 2012 and dropped to 24,231 cases in 2013. ${ }^{30,31}$ It is unclear if the resurgence is due to mutations in the B. pertussis bacteria, heightened awareness combined with improved diagnostic testing and increased reporting, or rapid waning of postacellular-vaccination immunity. ${ }^{32,33}$ While reported cases have increased in the general population, pertussis-related morbidity and mortality disproportionately affects infants younger than 12 months of age. ${ }^{34}$ Young infants are usually infected after exposure to a close contact, with $47-60 \%$ of cases due to exposure from infected parents. ${ }^{16}$ Adults with pertussis are often unaware of their diagnosis, given that many adults are either asymptomatic or have symptoms of a common cold.

Innovative strategies are needed to prevent infant pertussis given that infants do not have adequate vaccine-induced protection against pertussis until at least 6 months of age after 2 to 3 DTaP vaccine doses ${ }^{16}$ One strategy is to "cocoon" infants or decrease their risk of pertussis exposure by immunizing their close contacts against pertussis. In 2005, the CDC recommended that unvaccinated postpartum women and other close contacts of newborn 
infants receive the diphtheria and reduced tetanus toxoids and acellular pertussis vaccine (Tdap) to decrease infant exposure to pertussis. ${ }^{35}$ Despite some progress in Tdap administration during the immediate postpartum period, Tdap cocooning programs have not been successfully implemented. For example, new fathers and other close contacts are not routinely receiving Tdap. ${ }^{36}$ Moreover, it is unlikely that postpartum maternal immunization as a sole strategy is adequate to prevent infant pertussis. ${ }^{37}$ In October 2011, the CDC's Advisory Committee on Immunization Practices (ACIP), considered current safety data, the potential for protection against infant pertussis from transplacentally transferred maternal antibodies, and a decision analysis suggesting vaccination during pregnancy is superior to postpartum administration. During this meeting, the CDC changed their "cocooning" recommendation to administration of Tdap to unimmunized women during the late second or third trimester of pregnancy. Immediate postpartum Tdap vaccination should be offered to unimmunized women who did not receive Tdap during pregnancy. ${ }^{38,39}$ This was followed by a swift adoption of CDC recommendations by the American College of Obstetricians and Gynecologists (the College) in March 2012, demonstrating strong support by the College for interventions proven to benefit both mother and infant.

Very soon after ob-gyns began working to implement office processes to administer Tdap to unimmunized pregnant women in their practices, the CDC's ACIP voted unanimously in October 2012 to recommend Tdap vaccination to all pregnant women regardless of her prior history of Tdap receipt. ${ }^{40}$ Such aggressive action was taken due to the record-breaking numbers of pertussis cases and deaths, 48,277 and 20 in 2012, respectively. ${ }^{41}$ While passive $^{2}$ infant immunity has been documented after maternal immunization, ${ }^{42}$ limited, statistically nonsignificant data has been extrapolated to suggest that the optimal timing for Tdap is in the third trimester and at least 2 weeks prior to delivery to allow for an adequate maternal antibody response. While it is imperative that ob-gyns routinely administer Tdap to all pregnant women to avoid potentially devastating infant pertussis, many questions regarding vaccine effectiveness, optimal timing of vaccine administration, infant antibody correlates of protection, and safety of repeated close-interval dosing in multiparous women remain unanswered. Ongoing studies of Tdap vaccine in pregnancy will provide some of these answers. ${ }^{43,44}$

\section{Measles-Mumps-Rubella (MMR)}

Routine childhood vaccination and young adult booster dosing has dramatically diminished the incidence of measles, mumps and rubella in the United States. Measles is a paramyxovirus that presents routinely with rash, diarrhea, and otitis media along with bronchopneumonia or encephalitis in severe cases. Infection during pregnancy increases the risk of spontaneous abortion, preterm birth, and low birth weight. ${ }^{45}$ Mumps is another paramyxovirus that presents with flu-like symptoms and bilateral parotitis and is associated with spontaneous abortion. Rubella is a togavirus that presents with more nonspecific symptoms including lymphadenopathy, arthralgias, fever, and rash. Infection during pregnancy, especially in the first trimester, can be devastating. In the last U.S. rubella epidemic in 1964-1965, approximately 12.5 million rubella infections resulted in 11,250 spontaneous or therapeutic abortions, 2,100 neonatal deaths, and 20,000 infants born with 
congenital rubella syndrome. ${ }^{46}$ Serious sequelae of congenital rubella syndrome include deafness, cataracts, cardiac defects, neurologic damage, and death.

Although rubella cases dramatically declined over the last 40 years, inclusion of MMR in the universal vaccination program occurred in 2004, after which elimination of endemic rubella virus transmission was documented in the United States. ${ }^{46}$ From 2004-2012, 79 cases of rubella infection and six cases of congenital rubella syndrome were documented, with all sources being foreign import or unidentifiable. Three of the six congenital rubella syndrome cases occurred in 2012 among mothers who had been in Africa during early pregnancy. Thus, MMR vaccination continues to be of high relevance to obstetric care given the increasing immigration and global mobility of the U.S. population.

After two childhood MMR doses, college or graduate students should receive additional doses or have documented immunity against all three viruses. ${ }^{47}$ Given that MMR is a live attenuated vaccine and contraindicated in pregnancy, pregnancy status should be queried among all women of childbearing potential prior to vaccination with counseling to avoid conception for 4 weeks postvaccination. While the risk of fetal harm or vaccine-induced infection is theoretical, the potential severe consequence of infection during pregnancy outweighs the potential benefit of vaccination in the United States. The CDC Vaccine in Pregnancy Registry demonstrates no reported cases of congenital rubella syndrome after unintentional first-trimester vaccination. ${ }^{48}$ Thus, inadvertent MMR vaccination during pregnancy should not be considered grounds for pregnancy termination. ${ }^{48}$ Preconception screening and MMR administration is ideal for avoiding congenital rubella syndrome during the next future pregnancy rather than awaiting screening and postpartum administration. However, screening for rubella immunity should be included in the routine prenatal laboratory panel. All pregnant women who are susceptible to rubella should be vaccinated immediately postpartum, thus reducing or eliminating risk in subsequent pregnancies and infant exposure risk. ${ }^{47}$ Breastfeeding is not a contraindication for postpartum vaccination since any attenuated rubella virus excreted in breast milk and transmitted to the neonate results in asymptomatic infection. ${ }^{47}$

\section{Varicella}

Varicella-zoster virus (VZV), a member of the herpes virus family, causes chicken pox. Illness usually presents as a pruritic rash for 4-7 days, during which time the infected individual is highly contagious. ${ }^{49}$ Viral transmission occurs by direct contact with skin lesions or inhalation of aerosolized particles. Infection during pregnancy is associated with neonatal varicella or herpes zoster and congenital varicella syndrome, which is characterized by skin scarring, limb hypoplasia, low birth weight, and numerous other anomalies. ${ }^{50}$ Congenital varicella syndrome occurs in 1-2\% of cases of maternal varicella infection, with the greatest risk of occurrence associated with maternal infection from 13-20 weeks of gestation.

Varicella vaccine, a live attenuated vaccine, was first introduced in 1995 at which time there were more than 4 million cases, over 10,000 hospitalizations, and approximately 150 deaths per year in the United States. ${ }^{51}$ Ten years after national recommendations to administer a two-dose regimen as part of the routine childhood vaccination schedule, disease incidence 
has dropped by $90 \%$ with hospitalizations and deaths down by $71 \%$ and $97 \%$, respectively. In 2003-2004, there were eight reported deaths due to varicella, six of which occurred in children. Thus, vaccination is the most effective means of preventing varicella infection and congenital varicella syndrome. However, varicella vaccine is contraindicated in pregnancy due to its live attenuated formulation. Analogous to rubella vaccination in early pregnancy, no cases of congenital varicella syndrome have been reported after inadvertent varicella vaccination during pregnancy based on the VARIVAX Pregnancy Registry co-sponsored by Merck and Company, Inc., and the CDC. Therefore, the CDC recommends that unintentional vaccination in pregnancy does not imply a need for pregnancy termination. ${ }^{49,52}$ Moreover, preconception varicella vaccine administration is ideal to avoid congenital varicella syndrome during the next future pregnancy. Varicella immunity should be established for all pregnant women during early pregnancy by self-reported history of infection, history of vaccination, or documented serologic immunity. All pregnant women who are susceptible to VZV infection should be counseled about the risk of perinatal infection and instructed to immediately contact their provider in case of exposure. Documented exposure should be followed by prompt administration of varicella-zoster immunoglobulin. ${ }^{53}$ Antivirals such as acyclovir should be used for actual chicken pox infection with consideration of hospital observation for development of varicella-related complications such as respiratory disease. ${ }^{53}$ Mothers who are or suspected to be VZVsusceptible should receive two doses of vaccine postpartum - the first immediately postpartum and the second $4-8$ weeks later. ${ }^{49}$

\section{Vaccines Recommended in Pregnancy and Postpartum Based on Risk Factors and Special Circumstances (Table 1)}

\section{Hepatitis A}

Hepatitis A virus (HAV), a RNA picornavirus, causes fever, nausea, abdominal pain, and jaundice due to acute, self-limiting liver infection. HAV is transmitted through the fecal-oral route after close contact with infected individuals or contaminated food or drinks. ${ }^{54}$ First licensed in 1996, widespread HAV vaccination in the United States began in 1999. From 2007 to 2011, the number of HAV cases dropped from 2,979 to $1,398.55$ While HAV vaccine is included in the CDC childhood vaccination schedule at 12-23 months of age, $\mathrm{CDC}$ also recommends adult HAV vaccination for individuals deemed high risk for HAV exposure. Risk factors include travel to endemic areas, men having sex with men, exposure to individuals with HAV infection or those receiving clotting-factor concentrates due to clotting-factor deficiencies, and exposure to biological specimens. While there is not sufficient data to deem HAV vaccine safe for use during pregnancy, the current vaccine formulation is inactivated with no live virus components and thus extremely unlikely to cause infection or harm to mother, fetus, or infant. Pregnant women should receive HAV vaccine when the risk of infection outweighs the theoretical risk of administration of an inactivated vaccine. ${ }^{54}$

\section{Hepatitis B}

Hepatitis B virus (HBV), a DNA virus, causes acute liver infection with inflammation, vomiting, and jaundice. HBV can be self-limiting or achieve a chronic carrier state 
associated with long-term consequences including cirrhosis, liver cancer, liver failure, and death. HBV is transmitted after close contact with infected blood and bodily fluids. The current $\mathrm{HBV}$ vaccine is a recombinant DNA formulation based on the hepatitis B surface antigen ( $\mathrm{HBsAg}$ ) envelope protein. A three-vaccine series is highly effective for disease prevention, providing an indefinite protective antibody response in greater than $90 \%$ of vaccinated individual ${ }^{56} \mathrm{HBV}$ infections decreased $64 \%$ over the last decade, dropping from 8,036 cases in 2000 down to 2,890 cases in 2011 due to vaccine prevention. ${ }^{55}$

HBV during pregnancy, both the chronic carrier state as well as primary infection, is concerning for the risk of vertical transmission. Perinatally-acquired HBV infection is associated with the highest risk of developing chronic disease for the offspring. All pregnant women should be screened for hepatitis B surface antigen (HBsAg) status as part of standard prenatal laboratory testing. At-risk neonates should be treated with HB immunoglobulin (HBIg) prophylaxis and receive the first HBV vaccine dose within the first hours of life. ${ }^{57}$ Such a regimen is an effective strategy against peripartum transmission, which is the most common scenario. However, in utero transmission can and does occur, most likely in mothers who develop primary, acute HBV infection in late pregnancy. ${ }^{58}$ Therefore, HBV vaccination is the best preventive measure, including administration both preconception and during pregnancy. The three-dose HBV vaccine series should be initiated for pregnant women who have not been vaccinated previously and are at high risk of acquiring infection, namely those with one or more sexual partner in the past 6 months, history of a HBVpositive sexual partner or household contact, or intravenous drug use. ${ }^{56}$ Safety data, though somewhat limited, has not demonstrated any association with adverse maternal or fetal outcomes, consistent with the expected safety profile of a recombinant inactivated product that does not contain live virus. ${ }^{59}$ In addition to vaccination and HBIg, newly emerging data advocates for the use of antiviral therapy during the third trimester to prevent in utero transmission or prophylaxis failure. ${ }^{58}$ However, given the uncertain risks of antivirals during pregnancy, treatment is being considered only for pregnant women with advanced disease. Treatment may consist of lamivudine, telbivudine, or tenofovir but should only be considered in conjunction with a hepatology specialist.

\section{Pneumococcal Disease}

Streptococcus pneumoniae (pneumococcus) is a gram-positive bacterium associated with significant morbidity and mortality related to pneumonia, bacteremia, meningitis, and otitis media. ${ }^{60}$ About 900,000 U.S. individuals get pneumococcal pneumonia, resulting in 400,000 hospitalizations and 5-7\% mortality rate annually. There are 12,000 cases of bacteremia and 3,000 cases on meningitis, which carries a mortality rate of $10-15 \%$. Significant reductions in pneumococcal disease and related deaths occurred after inclusion of the seven serotype pneumococcal conjugate vaccines (PCV7) in the U.S. childhood vaccination schedule in 2000 (200,000 cases and 13,000 deaths prevented up to 2007). ${ }^{3}$ Further, the introduction of PCV13 in 2010 resulted in an additional 20,000 cases and 2,000 deaths averted. ${ }^{61}$ From 2010 to 2012 , there was an $88 \%$ reduction in childhood disease, which would be expected given that PCV is used exclusively in children. However, there was also a $47 \%$ reduction in adult pneumococcal disease, likely due to a reduction in exposure. Further, a broader 23serotype polysaccharide vaccine (PPSV23) is recommended for children and adults with 
chronic medical conditions and all adults at or after 65 years of age to prevent invasive pneumococcal disease. ${ }^{62}$ Risk factors include chronic heart disease, chronic lung disease including asthma, pre-existing diabetes, cigarette smoking, alcoholism, chronic liver disease, cerebrospinal fluid leaks, cochlear implants, congenital or acquired immunodeficiencies, diseases requiring immunosuppressant therapy, sickle cell disease and other hemoglobinopathies, and functional or anatomic asplenia.

Per the CDC, there is currently insufficient data to support routine administration of PCV13 or PPSV23 during pregnancy. ${ }^{18}$ A systematic review of six observational studies of PPSV23 in pregnancy demonstrated no increase in spontaneous abortion, teratogenicity, or preterm labor. ${ }^{59}$ Further, a randomized trial of 60 pregnant women receiving PPSV23 at 35 weeks of gestation demonstrated no safety concerns or adverse outcomes. ${ }^{63}$ Maternal PPSV23 administration results in protective maternal antibody titers for up to 12 months postpartum and significantly increases neonatal antibody titers at delivery. However, it is unknown if passive immunity results in disease protection for the infant. Per the CDC's adult immunization schedule, PPSV23 should be administered during pregnancy for women who have a medical risk factor(s), which likely affects a substantial number of women given the increasing incidence of obesity and related chronic conditions in the United States. ${ }^{18}$

\section{Meningococcal Disease}

Approximately 1,000 individuals in the United States will suffer from meningococcal disease (meningitis and sepsis) caused by an encapsulated bacterium called Neisseria. ${ }^{64}$ Despite the use of antibiotics, the mortality rate is $10-15 \%$, with up to $20 \%$ of survivors suffering significant sequelae including limb amputations, strokes, and neurocognitive abnormalities such as seizures, deafness, and severe cognitive impairment. There are two effective vaccines against meningococcal disease: tetravalent meningococcal conjugate vaccine (MCV4) and a tetravalent polysaccharide vaccine (MPSV4). In 2000, the CDC recommended MPSV4 prior to college entry based on risk. Although effective, MPSV4 does not confer long-lasting immunity as seen with MCV4. Therefore, in 2005, MCV4 was included in the U.S. childhood vaccination schedule as a 2-dose regimen for adolescents aged 11-18 years. However, there are many individuals who remain at high risk for the disease and thus should be vaccinated with MCV4 based on risk factors, ie, individuals living in close contact such as in dormitories or military barracks, individuals with complement deficiencies and functional or anatomic asplenia, researchers with routine exposure to Neisseria meningitidis isolates, and individuals living in hyperendemic areas. Based on available efficacy and safety data, MCV4 should be used through 55 years of age while MPSV4 is used after 55 years of age and for anyone at risk of exposure during an active disease outbreak.

Both MCV4 and MPSV4 are inactivated products and thus should not be associated with adverse maternal or fetal outcomes. However, the limited data that is available on meningococcal vaccination during pregnancy is using MPSV4 and not MCV4. A systematic review documented no association with maternal MPSV4 and teratogenesis, spontaneous abortion, or preterm birth. ${ }^{59}$ A study of third-trimester MPSV4 vaccination demonstrated an appropriate maternal antibody response but selective transplacental antibody transfer with 
rapid waning of infant immunity by 3 months of age. ${ }^{65}$ A recently completed trial of more than 4,000 pregnant Malian women receiving influenza vaccine or MPSV4 will provide much needed efficacy and safety data on MPSV4 in pregnancy. ${ }^{66}$ Given the lack of available safety data on MCV4, MPSV4 is recommended for use during pregnancy based on risk factors described above. ${ }^{18}$

\section{Travel Vaccinations}

Absent any specific medical or pregnancy-related contraindications, healthy pregnant women are able to travel safely without significant restrictions. The CDC recommends several vaccines for individuals traveling to areas with endemic vaccine-preventable diseases. ${ }^{67}$ Therefore, pregnant women planning international travel should be advised to search the CDC travel website, which provides up-to-date country-specific immunization recommendations and aids in risk factor determination. Three travel-related vaccinepreventable diseases that are frequently encountered are yellow fever, Japanese encephalitis, and typhoid fever.

Yellow fever, a mosquito-borne disease caused by an RNA flavivirus, is largely an asymptomatic infection. However, common symptoms are sudden onset of fever and headache with less common symptoms of photophobia, arthralgias, myalgias, vomiting, and epigastric pain. Severe infection is associated with multisystem organ failure, hemorrhage, and death. Yellow fever is endemic to tropical areas in South America and sub-Saharan Africa. Thus, live attenuated yellow fever vaccine is recommended for individuals planning travel to endemic areas and is often required prior to issuance of travel visas. ${ }^{67}$ Despite the general theoretical contraindication to live attenuated vaccines during pregnancy, yellow fever vaccine is the exception. While there is limited data on the efficacy and safety of yellow fever vaccine during pregnancy, one study which documented inadvertent vaccination of pregnant women during an outbreak demonstrated no association with teratogenesis, spontaneous abortion, or preterm birth. ${ }^{68}$ Therefore, the CDC recommends yellow fever vaccine during pregnancy if a woman must travel and her risk of exposure and infection is high enough (based on location, season, and activities planned during travel) to outweigh any potential theoretical risks of vaccination. ${ }^{69}$ Nonpregnant women of childbearing potential should be counseled to avoid conception for 4 weeks postvaccination.

Japanese encephalitis is another mosquito-borne RNA flavivirus that represents the most common vaccine-preventable disease cause of encephalitis in Asia. ${ }^{67}$ Less than $1 \%$ of infected individuals will manifest clinical symptoms of disease, with mild disease limited to fever or aseptic meningitis. However, serious illness presents with sudden onset of fever, headache, vomiting, and neurologic abnormalities including generalized weakness, movement disorders or acute paralysis, and seizures. While the mortality rate is $20-30 \%$, about $30-50 \%$ of survivors will have serious neurocognitive and psychiatric sequelae. An inactivated Japanese encephalitis vaccine was licensed for use in the U.S. in 2009 and recommended by the CDC for travelers who plan to spend 1 or more months in endemic areas or during disease outbreaks. Unfortunately there are no adequate studies of the vaccine during pregnancy. Due to the lack of adequate data, the CDC has not made a specific recommendation on the use of Japanese encephalitis vaccine during pregnancy. ${ }^{18}$ However, 
the CDC recommends that Japanese encephalitis vaccination should be considered for pregnant women planning longer-duration travel to endemic areas when the theoretical risk of immunization is outweighed by risk of infection. ${ }^{70}$

Typhoid fever is a life-threatening disease caused by the bacterium Salmonella typhi and is responsible for approximately 5,700 US cases each year. ${ }^{71}$ About $75 \%$ of cases are contracted during international travel as typhoid fever is quite common in the developing world and affects more than 21 million people annually. Infected individuals usually present with fever, fatigue, headache, and anorexia while severe disease is associated with intestinal hemorrhage and death. There are two vaccines against $S$. typhi currently available in the United States, which are a live attenuated oral vaccine and a polysaccharide vaccine, both of which are acceptable for adults traveling to endemic areas. Unfortunately, there are no data supporting the efficacy and safety of either vaccine in pregnancy. The CDC has made no formal, specific recommendation on the use of typhoid vaccines during pregnancy.

\section{Zoonotic Vaccine-Preventable Diseases}

Bacillus anthracis, a spore-forming bacterium, causes the zoonotic infection anthrax. Clinical manifestation depends on the exposure route (infected animal-to-animal tissue contact or bacterial spore exposure) and presents as cutaneous, injection (contaminated needle use), gastrointestinal, or inhalation anthrax with the latter two forms having high morbidity and mortality. ${ }^{72}$ Because $B$. anthracis spores can be aerosolized and remain viable for a long time, anthrax has been identified as a potentially serious and deadly biological weapon. The inactivated subunit anthrax vaccine adsorbed (AVA) is available in the United States. Pre-exposure vaccination is recommended by the CDC for individuals at high risk of exposure, ie military personnel, environmental investigators, emergency responders, and postal processing staff. However, pre-exposure AVA is not recommended during pregnancy given the low risk for exposure. Pregnancy is a Department of Defense exemption for preexposure prophylaxis, with vaccination recommended postpartum. For postexposure prophylaxis, individuals should be vaccinated with AVA followed by 60 days of antimicrobial therapy. ${ }^{72}$ While pre-exposure AVA is not routinely recommended, postexposure prophylaxis is recommended and should be administered to any pregnant woman with an anthrax exposure.

Contact with the saliva or central nervous system tissue of an individual or animal infected with rhabdovirus results in rabies infection. Rabies initially presents with flu-like symptoms followed by neurologic abnormalities and ultimately death if left untreated. Vaccination of household pets is the most effective method of human rabies prevention. ${ }^{73}$ There are two inactivated rabies vaccines available for human use in the United States and are recommended for individuals at high risk of exposure, ie, veterinarians, animal handlers, and travelers to endemic areas. Postexposure prophylaxis includes either rabies vaccine in conjunction with human rabies immunoglobulin (Ig), though human rabies Ig is not necessary in individuals who have been previously vaccinated. Limited studies have shown no association between maternal rabies vaccination and spontaneous abortion, teratogenesis, or preterm birth. ${ }^{74,75}$ Thus, the CDC recommends that post-exposure prophylaxis with vaccination and human rabies Ig should be administered to any pregnant woman after a 
moderate-risk or high-risk exposure to rabies. Pre-exposure prophylaxis in pregnancy can be considered if the risk of exposure is deemed high.

\section{Vaccines Currently Under Research and Development for Licensure for Maternal-Fetal Immunization}

To date, vaccines in the United States are not specifically licensed or targeted for use during pregnancy. However, vaccines aimed at fetal-infant immunization are in varying stages of research and development for which the target population will be pregnant women. Two examples are group B streptococcus (GBS) and respiratory syncytial virus (RSV). GBS is the leading cause of invasive infection during the first 90 days of life and is the predominate cause of neonatal sepsis and meningitis, even in the setting of intrapartum antibiotic prophylaxis. ${ }^{76}$ From 1993 to 2008, intrapartum antibiotic prophylaxis has reduced the incidence of early-onset neonatal GBS infection from 1.7 to 0.28 cases per 1,000 live births but has had no impact on late-onset GBS disease in the United States. ${ }^{77}$ Given the significant unmet need of late-onset disease ( $0.29-0.47$ per 1,000 live births) and evidence of protection in the setting of passive immunity, ${ }^{76,78}$ a GBS vaccine could be a more effective and reliable way to prevent both early- and late-onset disease. Moreover, a regimen of screening plus intrapartum antibiotic prophylaxis is neither feasible nor affordable in the developing world where invasive GBS disease remains a significant contributor to neonatal mortality and adverse pregnancy outcomes. A promising trivalent conjugated GBS vaccine is currently in phase II and III trials in pregnant women aimed at providing passive immunity to young infants. ${ }^{79}$

RSV is a RNA paramyxovirus named for how its surface F-proteins cause respiratory cell membranes to merge or form synctia. In the United States, $60 \%$ of infants will be infected during their first RSV season. ${ }^{80}$ Naturally-induced immunity wanes over time so that people can be repeatedly infected over the lifespan. Infection may be asymptomatic or similar to a cold but may cause severe disease in the elderly. RSV is the most common cause of bronchiolitis and pneumonia during the first year of life, often necessitating hospitalization and resulting in recurrent wheezing. Given that infection with this ubiquitous virus is unavoidable, a preventive vaccine would be ideal. A formalin-inactivated vaccine was developed and studied in the 1960s but was not efficacious and was associated with enhanced disease among vaccinated children. ${ }^{81}$ At present, the only preventive therapy available is palivizumab, an effective yet costly monoclonal antibody that is given as monthly injections during the RSV season. Given the significant cost and need for repeated dosing, palivizumab is restricted for use in high-risk infants, namely preterm infants, those with bronchopulmonary dysplasia, or those born with congenital heart or airway defects. A recombinant RSV vaccine is currently in phase II clinical and dose-ranging trials in nonpregnant women, with plans for a phase I trial in pregnant women in the near future. ${ }^{82}$

\section{Barriers to Vaccination in Pregnancy and Patient-Provider Resources}

Although national vaccination programs have led to significant declines in vaccinepreventable diseases in the United States, meeting the Healthy People 2020 objectives for adult vaccination remains a daunting task. Acceptance of vaccination during pregnancy is

Obstet Gynecol. Author manuscript; available in PMC 2016 January 01. 
affected by questions of maternal-fetal safety. Qualitative research involving patients and providers suggests that common perceived barriers include fear of adverse pregnancy outcomes, fear of vaccine-transmitted infection, lack of awareness of national recommendations, lack of provider recommendation, inconvenience of vaccination, and concerns about insurance coverage. ${ }^{83,84}$ For progress to occur, it is imperative that ob-gyns play an active role in overcoming such barriers, as evidenced by pregnant women being 5 to 50 more likely to accept a vaccine if directly recommended by their provider. ${ }^{85-87}$ To aid ob-gyns and patients, the College publishes and maintains up-to-date Committee Opinions on specific vaccines and guidance on implementing vaccination processes into ObGyn practice. In 2011, the College launched the Immunization for Women website, which provides up-to-date information on vaccine recommendations, safety, and hyperlinks to ACOG Committee Opinions, educational materials, and frequently asked questions, further demonstrating the College's commitment to immunization of women across the lifespan. ${ }^{88}$ Immunizationforwomen.org also provides hyperlinks to numerous highly informative, userfriendly resources such as the CDC, Immunization Action Coalition, and the National Vaccine Program Office.

Specifically regarding vaccine safety, there has been much controversy over possible associations between vaccines and complicated, multietiological health outcomes, such as autism spectrum disorders in children. Research to date involving more than 100,000 children that has been vetted by the Institute of Medicine, CDC, World Health Organization, American Academy of Pediatrics and numerous other agencies has very convincingly shown that there is no such association between vaccines and autism. ${ }^{89,90}$ On the contrary, these studies have further supported the safety and effectiveness of vaccines in reducing infectious disease and improving overall health. Continued research on the overall health effects and safety of vaccines is warranted.

Further, ob-gyns who are concerned about potential liability related to vaccination providers can be reassured by the protection provided through the National Childhood Vaccine Injury Act (NCVIA), which covers all vaccines that are included in the childhood vaccination schedule regardless of who actually receives the vaccine, including adults and pregnant women. To aid all providers in safely administering vaccines, the CDC maintains a Vaccine Information Statement (VIS) for every vaccine licensed for administration in the United States. A VIS informs providers and vaccinees about the benefits and risks of a specific vaccine and is required by the NCVIA to be given to all vaccinees (or their parent or legal representative) prior to vaccination. Ob-gyns and nurses should be familiar with the information contained in the VISs for any vaccines administered in their office so that they can effectively screen and counsel their patients on any vaccine contraindications, risks, and benefits. Ob-gyns should be aware of additional tools in the event that an adverse event does occur in a pregnant woman, which are the Vaccine Adverse Event Reporting System (VAERS) and the Clinical Immunization Safety Assessment Project (CISA). Co-sponsored by the CDC and the U.S. Food and Drug Administration, VAERS is a national postmarketing national vaccine safety and side effect surveillance program initiated after NCVIA. ${ }^{91}$ Anyone can submit a VAERS report, as it is considered a public health entity and does not require individual authorization per HIPAA criteria. With over 200,000 reports since its inception in 1990 (mostly mild side effects), VAERS has demonstrated its public 
health importance by identifying risks that are small yet higher than chance alone, thus guiding national recommendations. Sponsored by the CDC's Immunization Safety Office, CISA provides a free-of-charge clinical evaluation service of an adverse event that is unexpected or not explainable by available data. ${ }^{92}$ After obtaining all pertinent medical records, reviewing medical literature and VAERS, vaccine safety experts from the CDC and the CISA academic medical centers review the clinical case in conjunction with the reporting provider. Advice garnered from CISA consultation is to be used in further decision-making regarding future vaccination rather than immediate patient care.

\section{Conclusion}

Vaccination during pregnancy is a vital preventive measure in routine obstetric care, serving to protect mother, fetus, and infant. Influenza and Tdap vaccines are specifically recommended for all pregnant women while others recommended for postpartum administration (MMR and varicella) or depending upon risk factors (hepatitis A and B, pneumococcal and meningococcal vaccines). In theory, inactivated vaccines should be safe for use during pregnancy but specific studies or data on use during pregnancy is limited for most vaccines. Thus, it is essential that future studies on vaccines in pregnancy focus on immunogenicity and safety for mother and infant and the potential for not only maternal but also direct fetal and infant benefit. In light of the perceived and actual barriers to increasing adult vaccine coverage and the health consequences of vaccine-preventable diseases for pregnant women and young infants, ob-gyns must take an active role in educating and administering vaccines to pregnant women.

\section{Supplementary Material}

Refer to Web version on PubMed Central for supplementary material.

\section{References}

1. Centers for Disease Control and Prevention. [Accessed March 17, 2014] Vaccines and Immunizations - Vaccines and Preventable Diseases. http://www.cdc.gov/vaccines/vpd-vac/

2. Centers for Disease Control and Prevention. Ten great public health achievements--United States, 2001-2010. MMWR. Morbidity and mortality weekly report. May 20; 2011 60(19):619-623. [PubMed: 21597455]

3. National Foundation for Infectious Diseases. [Accessed December 30, 2013] Facts About Adult Immunization 2009. http://www.nfid.org/publications/factsheets/adultfact.pdf

4. US Dept of Health and Human Services. Topics \& Objectives: Immunization and Infectious Diseases. 2020.

5. Centers for Disease Control and Prevention. Noninfluenza vaccination coverage among adults United States, 2011. MMWR. Morbidity and mortality weekly report. Feb 1; 2013 62(4):66-72. [PubMed: 23364272]

6. Increasing Appropriate Vaccination. [Accessed January 3, 2014] http:// www.thecommunityguide.org/vaccines/index.html

7. Vaccines for the 21st Century: A Tool for Decisionmaking. The National Academies Press; 2000.

8. Leader S, Perales PJ. Provision of primary-preventive health care services by obstetriciangynecologists. Obstetrics and gynecology. Mar; 1995 85(3):391-395. [PubMed: 7862378] 
9. Centers for Disease Control and Prevention. Maternal and Infant outcomes amongs severely ill pregnant and postpartum women with 2009 pandemic influenza A (H1N1)--United States, April 2009-August 2010. MMWR Morbidity and mortality weely report. 2011; 60(35):1193-1196.

10. Haberg SE, Trogstad L, Gunnes N, et al. Risk of fetal death after pandemic influenza virus infection or vaccination. The New England journal of medicine. Jan 24; 2013 368(4):333-340. [PubMed: 23323868]

11. Louie JK, Acosta M, Jamieson DJ, Honein MA. Severe 2009 H1N1 influenza in pregnant and postpartum women in California. The New England journal of medicine. Jan 7; 2010 362(1):2735. [PubMed: 20032319]

12. Neuzil KM, Reed GW, Mitchel EF, Simonsen L, Griffin MR. Impact of influenza on acute cardiopulmonary hospitalizations in pregnant women. American journal of epidemiology. Dec 1; 1998 148(11):1094-1102. [PubMed: 9850132]

13. Pierce M, Kurinczuk JJ, Spark P, Brocklehurst P, Knight M, Ukoss. Perinatal outcomes after maternal 2009/H1N1 infection: national cohort study. Bmj. 2011; 342:d3214. [PubMed: 21672992]

14. Zaman K, Roy E, Arifeen SE, et al. Effectiveness of maternal influenza immunization in mothers and infants. The New England journal of medicine. Oct 9; 2008 359(15):1555-1564. [PubMed: 18799552]

15. Englund JAMI, Hammill H. Maternal Immunization with Influenza or Tetanus Toxoid Vaccine for Passive Antibody Protection in Young Infants. JID. 1993; 168:647-656. [PubMed: 8354906]

16. Edwards KM. Overview of pertussis: focus on epidemiology, sources of infection, and long term protection after infant vaccination. The Pediatric infectious disease journal. Jun; 2005 24(6 Suppl):S104-108. [PubMed: 15931137]

17. Centers for Disease Control and Prevention. Influenza vaccination coverage among pregnant women--United States, 2012-13 influenza season. MMWR. Morbidity and mortality weekly report. Sep 27; 2013 62(38):787-792. [PubMed: 24067583]

18. Centers for Disease Control and Prevention. Guidelines for Vaccinating Pregnant Women. http:// www.cdc.gov/vaccines/pubs/preg-guide.htm\#ppsv23

19. The History of Vaccines - Different Types of Vaccines. http://www.historyofvaccines.org/content/ articles/different-types-vaccines

20. Plotkin, SOW.; Offit, P. Vaccines. 6. Philadelphia, PA: Saunders; 2012.

21. Rasmussen SA, Jamieson DJ, Bresee JS. Pandemic influenza and pregnant women. Emerging infectious diseases. Jan; 2008 14(1):95-100. [PubMed: 18258087]

22. Cantu J, Tita AT. Management of influenza in pregnancy. American journal of perinatology. Feb; 2013 30(2):99-103. [PubMed: 23271379]

23. Yates L, Pierce M, Stephens S, et al. Influenza A/H1N1v in pregnancy: an investigation of the characteristics and management of affected women and the relationship to pregnancy outcomes for mother and infant. Health technology assessment. Jul; 2010 14(34):109-182. [PubMed: 20630123]

24. Toback SL, Beigi R, Tennis P, Sifakis F, Calingaert B, Ambrose CS. Maternal outcomes among pregnant women receiving live attenuated influenza vaccine. Influenza and other respiratory viruses. Jan; 2012 6(1):44-51. [PubMed: 21672166]

25. Benowitz I, Esposito DB, Gracey KD, Shapiro ED, Vazquez M. Influenza vaccine given to pregnant women reduces hospitalization due to influenza in their infants. Clinical infectious diseases: an official publication of the Infectious Diseases Society of America. Dec 15; 2010 51(12):1355-1361. [PubMed: 21058908]

26. Poehling KA, Szilagyi PG, Staat MA, et al. Impact of maternal immunization on influenza hospitalizations in infants. American journal of obstetrics and gynecology. Jun; 2011 204(6 Suppl 1):S141-148. [PubMed: 21492825]

27. Harper SA, Bradley JS, Englund JA, et al. Seasonal influenza in adults and children--diagnosis, treatment, chemoprophylaxis, and institutional outbreak management: clinical practice guidelines of the Infectious Diseases Society of America. Clinical infectious diseases: an official publication of the Infectious Diseases Society of America. Apr 15; 2009 48(8):1003-1032. [PubMed: 19281331] 
28. Centers for Disease Control and Prevention. [Accessed March 18, 2014] Influenza Antiviral Medications: Summary for Clinicians. http://www.cdc.gov/flu/professionals/antivirals/summaryclinicians.htm

29. Tanaka T, Nakajima K, Murashima A, Garcia-Bournissen F, Koren G, Ito S. Safety of neuraminidase inhibitors against novel influenza $\mathrm{A}(\mathrm{H} 1 \mathrm{~N} 1)$ in pregnant and breastfeeding women. CMAJ: Canadian Medical Association journal = journal de l'Association medicale canadienne. Jul 7; 2009 181(1-2):55-58.

30. Centers for Disease Control and Prevention. Notifiable Diseases and Mortality Tables. Atlanta, Ga: Centers for Disease Control \& Prevention; [January 3, 2014 2014]

31. Centers for Disease Control and Prevention. [Accessed January 3, 2014] Pertussis (Whooping Cough) Outbreaks. http://www.cdc.gov/pertussis/outbreaks/index.html

32. Weber C, Boursaux-Eude C, Coralie G, Caro V, Guiso N. Polymorphism of Bordetella pertussis isolates circulating for the last 10 years in France, where a single effective whole-cell vaccine has been used for more than 30 years. Journal of clinical microbiology. Dec; 2001 39(12):4396-4403. [PubMed: 11724851]

33. Wendelboe AM, Van Rie A, Salmaso S, Englund JA. Duration of immunity against pertussis after natural infection or vaccination. The Pediatric infectious disease journal. May; 2005 24(5 Suppl):S58-61. [PubMed: 15876927]

34. Vitek CR, Pascual FB, Baughman AL, Murphy TV. Increase in deaths from pertussis among young infants in the United States in the 1990s. The Pediatric infectious disease journal. Jul; 2003 22(7):628-634. [PubMed: 12867839]

35. Kretsinger K, Broder KR, Cortese MM, et al. Preventing tetanus, diphtheria, and pertussis among adults: use of tetanus toxoid, reduced diphtheria toxoid and acellular pertussis vaccine recommendations of the Advisory Committee on Immunization Practices (ACIP) and recommendation of ACIP, supported by the Healthcare Infection Control Practices Advisory Committee (HICPAC), for use of Tdap among health-care personnel. MMWR. Recommendations and reports: Morbidity and mortality weekly report. Recommendations and reports/Centers for Disease Control. Dec 15; 2006 55(RR-17):1-37.

36. Steiner B, Swamy GK, Walter EB. Engaging Expectant Parents to Receive Tdap Vaccination. American journal of perinatology. Aug 5.2013

37. Castagnini LA, Healy CM, Rench MA, Wootton SH, Munoz FM, Baker CJ. Impact of maternal postpartum tetanus and diphtheria toxoids and acellular pertussis immunization on infant pertussis infection. Clinical infectious diseases: an official publication of the Infectious Diseases Society of America. Jan 1; 2012 54(1):78-84. [PubMed: 22075790]

38. Centers for Disease Control and Prevention. Updated Recommendations for Use of Tetanus Toxoid, Reduced Diphtheria Toxoid and Acellular Pertussis Vaccine (Tdap) in Pregnant Women and Persons Who Have or Anticipate Having Close Contact with an Infant Aged <12 Months Advisory Committee on Immunization Practices (ACIP). 2011; 60:1424-1426.

39. Terranella A, Asay GR, Messonnier ML, Clark TA, Liang JL. Pregnancy dose Tdap and postpartum cocooning to prevent infant pertussis: a decision analysis. Pediatrics. Jun; 2013 131(6):e1748-1756. [PubMed: 23713104]

40. Centers for Disease Control and Prevention. Updated Recommendations for Use of Tetanus Toxoid, Reduced Diphtheria Toxoid, and Acellular Pertussis Vaccine (Tdap) in Pregnant Women — Advisory Committee on Immunization Practices (ACIP). 2012; 622013:131-135.

41. Centers for Disease Control and Prevention. [Accessed January 3, 2013] 2012 Final Pertussis Surveillance Report. 2013. http://www.cdc.gov/pertussis/downloads/pertussis-surveillancereport.pdf

42. Gall SA, Myers J, Pichichero M. Maternal immunization with tetanus-diphtheria-pertussis vaccine: effect on maternal and neonatal serum antibody levels. American journal of obstetrics and gynecology. Apr; 2011 204(4):334, e331-335. [PubMed: 21272845]

43. [Accessed January 3, 2014] Pertussis Vaccine in Healthy Pregnant Women. http:// clinicaltrials.gov/show/nct00707148

44. [Accessed January 3, 2014] Pertussis Maternal Immunization Study. http://clinicaltrials.gov/show/ nct00553228 
45. Siegel M, Fuerst HT. Low birth weight and maternal virus diseases. A prospective study of rubella, measles, mumps, chickenpox, and hepatitis. JAMA: the journal of the American Medical Association. Aug 29; 1966 197(9):680-684.

46. Centers for Disease Control and Prevention. Three cases of congenital rubella syndrome in the postelimination era--Maryland, Alabama, and Illinois, 2012. MMWR. Morbidity and mortality weekly report. Mar 29; 2013 62(12):226-229. [PubMed: 23535689]

47. Watson JC, Hadler SC, Dykewicz CA, Reef S, Phillips L. Measles, mumps, and rubella--vaccine use and strategies for elimination of measles, rubella, and congenital rubella syndrome and control of mumps: recommendations of the Advisory Committee on Immunization Practices (ACIP). MMWR. Recommendations and reports: Morbidity and mortality weekly report.

Recommendations and reports/Centers for Disease Control. May 22; 1998 47(RR-8):1-57. [PubMed: 9639369]

48. Centers for Disease Control and Prevention. Revised ACIP recommendation for avoiding pregnancy after receiving a rubella-containing vaccine. MMWR. Morbidity and mortality weekly report. Dec 14.2001 50(49):1117. [PubMed: 11794623]

49. Marin M, Guris D, Chaves SS, et al. Prevention of varicella: recommendations of the Advisory Committee on Immunization Practices (ACIP). MMWR. Recommendations and reports: Morbidity and mortality weekly report. Recommendations and reports/Centers for Disease Control. Jun 22; 2007 56(RR-4):1-40. [PubMed: 17585291]

50. Enders G, Miller E, Cradock-Watson J, Bolley I, Ridehalgh M. Consequences of varicella and herpes zoster in pregnancy: prospective study of 1739 cases. Lancet. Jun 18; 1994 343(8912): 1548-1551. [PubMed: 7802767]

51. Centers for Disease Control and Prevention. [Accessed January 3, 2014] Monitoring the Impact of Varicella Vaccination. http://www.cdc.gov/chickenpox/hcp/monitoring-varicella.html

52. [Accessed January 3, 2014] Merck Pregnancy Registries: Varicella Zoster Virus-Containing Vaccines. http://www.merckpregnancyregistries.com/varivax.html

53. ACOG practice bulletin. Perinatal viral and parasitic infections. Number 20, September 2000. (Replaces educational bulletin number 177, February 1993). American College of Obstetrics and Gynecologists. International journal of gynaecology and obstetrics: the official organ of the International Federation of Gynaecology and Obstetrics. Jan; 2002 76(1):95-107.

54. Fiore AE, Wasley A, Bell BP. Prevention of hepatitis A through active or passive immunization: recommendations of the Advisory Committee on Immunization Practices (ACIP). MMWR. Recommendations and reports: Morbidity and mortality weekly report. Recommendations and reports/Centers for Disease Control. May 19; 2006 55(RR-7):1-23.

55. Centers for Disease Control and Prevention. [Accessed January 3, 2014] Surveillance for Viral Hepatitis- United States, 2011. http://www.cdc.gov/hepatitis/Statistics/2011Surveillance/

56. Mast EE, Weinbaum CM, Fiore AE, et al. A comprehensive immunization strategy to eliminate transmission of hepatitis B virus infection in the United States: recommendations of the Advisory Committee on Immunization Practices (ACIP) Part II: immunization of adults. MMWR. Recommendations and reports: Morbidity and mortality weekly report. Recommendations and reports/Centers for Disease Control. Dec 8; 2006 55(RR-16):1-33. quiz CE31-34. [PubMed: 17159833]

57. American College of Obstetricians and Gynecologists. ACOG Practice Bulletin No. 86: Viral hepatitis in pregnancy. Obstetrics and gynecology. Oct; 2007 110(4):941-956. [PubMed: 17906043]

58. Pan CQ, Lee HM. Antiviral therapy for chronic hepatitis B in pregnancy. Seminars in liver disease. May; 2013 33(2):138-146. [PubMed: 23749670]

59. Makris MC, Polyzos KA, Mavros MN, Athanasiou S, Rafailidis PI, Falagas ME. Safety of hepatitis B, pneumococcal polysaccharide and meningococcal polysaccharide vaccines in pregnancy: a systematic review. Drug safety: an international journal of medical toxicology and drug experience. Jan 1; 2012 35(1):1-14.

60. Centers for Disease Control and Prevention. [Accessed January 3, 2014] Pneumococcal Disease. http://www.cdc.gov/pneumococcal/index.html 
61. Moore, M. Update on Effectiveness and Impact of PCV13 use among U.S. Children. Paper presented at: Advisory Committee on Immunization Practice; October 23, 2013, 2013; Atlanta, GA.

62. Centers for Disease Control and Prevention. Updated recommendations for prevention of invasive pneumococcal disease among adults using the 23-valent pneumococcal polysaccharide vaccine (PPSV23). MMWR. Morbidity and mortality weekly report. Sep 3; 2010 59(34):1102-1106. [PubMed: 20814406]

63. Munoz FM, Englund JA, Cheesman CC, et al. Maternal immunization with pneumococcal polysaccharide vaccine in the third trimester of gestation. Vaccine. Dec 12; 2001 20(5-6):826837. [PubMed: 11738746]

64. Cohn AC, MacNeil JR, Clark TA, et al. Prevention and control of meningococcal disease: recommendations of the Advisory Committee on Immunization Practices (ACIP). MMWR. Recommendations and reports: Morbidity and mortality weekly report. Recommendations and reports/Centers for Disease Control. Mar 22; 2013 62(RR-2):1-28. [PubMed: 23515099]

65. O’Dempsey TJ, McArdle T, Ceesay SJ, et al. Meningococcal antibody titres in infants of women immunised with meningococcal polysaccharide vaccine during pregnancy. Archives of disease in childhood. Fetal and neonatal edition. Jan; 1996 74(1):F43-46. [PubMed: 8653435]

66. [Accessed January 3, 2014] Maternal Flu Vaccine Trial in Bamako, Mali. http://clinicaltrials.gov/ show/NCT01430689

67. Centers for Disease Control and Prevention. CDC Health Information for International Travel. Oxford University Press; 2014.

68. Suzano CE, Amaral E, Sato HK, Papaiordanou PM. Campinas Group on Yellow Fever Immunization during P. The effects of yellow fever immunization (17DD) inadvertently used in early pregnancy during a mass campaign in Brazil. Vaccine. Feb 27; 2006 24(9):1421-1426. [PubMed: 16236402]

69. Staples JE, Gershman M, Fischer M. Centers for Disease C and Prevention. Yellow fever vaccine: recommendations of the Advisory Committee on Immunization Practices (ACIP). MMWR. Recommendations and reports: Morbidity and mortality weekly report. Recommendations and reports/Centers for Disease Control. Jul 30; 2010 59(RR-7):1-27.

70. Fischer M, Lindsey N, Staples JE, Hills S. Centers for Disease C and Prevention. Japanese encephalitis vaccines: recommendations of the Advisory Committee on Immunization Practices (ACIP). MMWR. Recommendations and reports: Morbidity and mortality weekly report. Recommendations and reports/Centers for Disease Control. Mar 12; 2010 59(RR-1):1-27.

71. Centers for Disease Control and Prevention. [Accessed January 3, 2014] National Center for Emerging and Zoonotic Infectious Diseases, Typhoid Fever. http://www.cdc.gov/nczved/divisions/ dfbmd/diseases/typhoid_fever/

72. Wright JG, Quinn CP, Shadomy S, Messonnier N. Centers for Disease C and Prevention. Use of anthrax vaccine in the United States: recommendations of the Advisory Committee on Immunization Practices (ACIP), 2009. MMWR. Recommendations and reports: Morbidity and mortality weekly report. Recommendations and reports/Centers for Disease Control. Jul 23; 2010 59(RR-6):1-30. [PubMed: 20651644]

73. Manning SE, Rupprecht CE, Fishbein D, et al. Human rabies prevention--United States, 2008: recommendations of the Advisory Committee on Immunization Practices. MMWR. Recommendations and reports: Morbidity and mortality weekly report. Recommendations and reports/Centers for Disease Control. May 23; 2008 57(RR-3):1-28. [PubMed: 18496505]

74. Chutivongse S, Wilde H, Benjavongkulchai M, Chomchey P, Punthawong S. Postexposure rabies vaccination during pregnancy: effect on 202 women and their infants. Clinical infectious diseases: an official publication of the Infectious Diseases Society of America. Apr; 1995 20(4):818-820. [PubMed: 7795079]

75. Rupprecht CE, Briggs D, Brown CM, et al. Use of a reduced (4-dose) vaccine schedule for postexposure prophylaxis to prevent human rabies: recommendations of the advisory committee on immunization practices. MMWR. Recommendations and reports: Morbidity and mortality weekly report. Recommendations and reports/Centers for Disease Control. Mar 19; 2010 59(RR-2):1-9. [PubMed: 20300058] 
76. Verani JR, McGee L, Schrag SJ. Division of Bacterial Diseases NCfI, Respiratory Diseases CfDC, Prevention. Prevention of perinatal group B streptococcal disease--revised guidelines from CDC, 2010. MMWR. Recommendations and reports: Morbidity and mortality weekly report.

Recommendations and reports/Centers for Disease Control. Nov 19; 2010 59(RR-10):1-36. [PubMed: 21088663]

77. Centers for Disease Control and Prevention. [Accessed January 3, 2014] Group B Strep (GBS). http://www.cdc.gov/groupbstrep/clinical-overview.html

78. Jordan HT, Farley MM, Craig A, et al. Revisiting the need for vaccine prevention of late-onset neonatal group B streptococcal disease: a multistate, population-based analysis. The Pediatric infectious disease journal. Dec; 2008 27(12):1057-1064. [PubMed: 18989238]

79. [Accessed January 2, 2014] Immune Response Induced by a Vaccine Against Group B Streptococcus and Safety in Pregnant Women and Their Offsprings. http://clinicaltrials.gov/show/ NCT01446289

80. Centers for Disease Control and Prevention. RSV Trends and Surveillance. http:// www.cdc.gov/rsv/research/us-surveillance.html

81. Kim HW, Canchola JG, Brandt CD, et al. Respiratory syncytial virus disease in infants despite prior administration of antigenic inactivated vaccine. American journal of epidemiology. Apr; 1969 89(4):422-434. [PubMed: 4305198]

82. [Accessed January 2, 2014] Safety Study of Respiratory Syncytial Virus (RSV)-Fusion (F) Protein Particle Vaccine. http://clinicaltrials.gov/show/NCT01290419

83. Leddy MA, Anderson BL, Power ML, Gall S, Gonik B, Schulkin J. Changes in and current status of obstetrician-gynecologists' knowledge, attitudes, and practice regarding immunization. Obstetrical \& gynecological survey. Dec; 2009 64(12):823-829. [PubMed: 19939296]

84. Panda B, Stiller R, Panda A. Influenza vaccination during pregnancy and factors for lacking compliance with current CDC guidelines. The journal of maternal-fetal \& neonatal medicine: the official journal of the European Association of Perinatal Medicine, the Federation of Asia and Oceania Perinatal Societies, the International Society of Perinatal Obstet. Mar; 2011 24(3):402406.

85. Ahluwalia IB, Jamieson DJ, Rasmussen SA, D'Angelo D, Goodman D, Kim H. Correlates of seasonal influenza vaccine coverage among pregnant women in Georgia and Rhode Island. Obstetrics and gynecology. Oct; 2010 116(4):949-955. [PubMed: 20859160]

86. American College of Obstetricians and Gynecologists. Committee opinion no. 608: influenza vaccination during pregnancy. Obstetrics and gynecology. Sep; 2014 124(3):648-651. [PubMed: 25162283]

87. Shavell VI, Moniz MH, Gonik B, Beigi RH. Influenza immunization in pregnancy: overcoming patient and health care provider barriers. American journal of obstetrics and gynecology. Sep; 2012 207(3 Suppl):S67-74. [PubMed: 22920063]

88. [Accessed December 27, 2013] Immunization for Women: Immunization Information for Ob-Gyns and Their Patients. http://www.immunizationforwomen.org/

89. American Academy of Pediatrics. [Accessed December 27, 2013] Vaccine Safety: Examine the Evidence. 2013. http://www2.aap.org/immunization/families/faq/VaccineStudies.pdf

90. Immunization Action Coalition. [Accessed January 3, 2014] Evidence Shows Vaccines Unrelated to Autism. http://www.immunize.org/catg.d/p4028.pdf

91. [Accessed January 3, 2014] Vaccine Adverse Event Reporting System (VAERS). https:// vaers.hhs.gov/index

92. [Accessed January 3, 2014] Vaccine Safety, CISA Evaluation. http://www.cdc.gov/vaccinesafety/ Activities/cisa/cisa-evaluation.html 
Table 1

Current Vaccine Recommendations for Pregnant Women

\begin{tabular}{|c|c|c|c|}
\hline Vaccine & Vaccine Type & Pregnancy Recommendation & General Adult Recommendation \\
\hline \multicolumn{4}{|c|}{ Vaccines Recommended for All Pregnant Women } \\
\hline Influenza & $\begin{array}{l}\text { Inactivated viral subunit or live- } \\
\text { attenuated viral recombinant }\end{array}$ & $\begin{array}{l}1 \text { dose of inactivated vaccine } \\
\text { administered during flu season, } \\
\text { any gestational age }\end{array}$ & $\begin{array}{l}1 \text { dose of inactivated or live- } \\
\text { attenuated vaccine administered } \\
\text { annually during flu season }\end{array}$ \\
\hline $\begin{array}{l}\text { Tetanus Diphtheria } \\
\text { Pertussis (Tdap/Td) }\end{array}$ & $\begin{array}{l}\text { Tetanus \& Diphtheria - Inactivated } \\
\text { toxoids; Acellular pertussis - } \\
\text { Inactivated subunit }\end{array}$ & $\begin{array}{l}1 \text { dose Tdap after } 20 \text { weeks, } \\
\text { preferably } \sim 28 \text { weeks, } \\
\text { regardless of prior Tdap receipt }\end{array}$ & $\begin{array}{l}\text { Substitute } 1 \text { lifetime dose of Tdap } \\
\text { for Td booster; return to Td booster } \\
\text { every } 10 \text { years or sooner if } \\
\text { exposure occurs }\end{array}$ \\
\hline \multicolumn{4}{|c|}{ Vaccines Recommended for Postpartum Women (Contraindicated During Pregnancy) } \\
\hline $\begin{array}{l}\text { Measles Mumps Rubella } \\
\text { (MMR) }\end{array}$ & Live-attenuated viral & $\begin{array}{l}1 \text { dose immediately postpartum } \\
\text { for if rubella non-immune or } \\
\text { equivocal }\end{array}$ & $\begin{array}{l}1-2 \text { doses, lifetime; additional } 1 \\
\text { dose }>55 \text { if risk factor present }\end{array}$ \\
\hline Varicella & Live-attenuated (viral) & $\begin{array}{l}1 \text { dose immediately if varicella } \\
\text { non-immune }\end{array}$ & 2 doses, lifetime \\
\hline \multicolumn{4}{|c|}{ Vaccines Recommended for Pregnant Women with Risk Factors/Special Circumstances } \\
\hline Hepatitis A & Inactivated whole-cell viral & $\begin{array}{l}2 \text { doses if risk of infection } \\
\text { outweighs theoretical risk of } \\
\text { vaccine }\end{array}$ & 2 lifetime doses \\
\hline Hepatitis B & Inactivated viral recombinant subunit & $\begin{array}{l}3 \text { doses if previously } \\
\text { unvaccinated or at high risk of } \\
\text { exposure }\end{array}$ & 3 lifetime doses \\
\hline Pneumococcal (PPSV23) & Inactivated bacterial polysaccharide & 1 dose if risk factor present & $\begin{array}{l}1-2 \text { doses if risk factor present; } 1 \\
\text { dose for all individuals } \ 65 \text { years }\end{array}$ \\
\hline Meningococcal (MPSV4) & Inactivated bacterial polysaccharide & 1 dose if risk factor present & $\begin{array}{l}\text { Can be used for children }<2 \text { years } \\
\text { and adults }>55 \text { years and during } \\
\text { epidemics }\end{array}$ \\
\hline Yellow fever & Live-attenuated viral & $\begin{array}{l}1 \text { dose if travel to endemic } \\
\text { regions and risk of infection } \\
\text { outweighs theoretical risk of } \\
\text { vaccine }\end{array}$ & 1 dose for travel to endemic regions \\
\hline Japanese Encephalitis & Live-attenuated viral & $\begin{array}{l}1 \text { dose if travel to endemic } \\
\text { regions and risk of infection } \\
\text { outweighs theoretical risk of } \\
\text { vaccine }\end{array}$ & 1 dose for travel to endemic regions \\
\hline Typhoid & Live-attenuated bacterial recombinant & $\begin{array}{l}\text { Not recommended due to lack } \\
\text { of data }\end{array}$ & For travel to endemic regions \\
\hline Anthrax & Inactivated subunit & $\begin{array}{l}\text { Post-exposure prophylaxis for } \\
\text { all pregnant women; pre- } \\
\text { exposure prophylaxis is not } \\
\text { recommended due to lack of } \\
\text { data }\end{array}$ & $\begin{array}{l}\text { Pre-exposure prophylaxis if risk } \\
\text { factor; post-exposure prophylaxis } \\
\text { for all adults }\end{array}$ \\
\hline Rabies & Inactivated whole-cell viral & $\begin{array}{l}\text { Post-exposure prophylaxis; } \\
\text { consider pre-exposure } \\
\text { prophylaxis if risk of exposure } \\
\text { is very high }\end{array}$ & $\begin{array}{l}\text { Pre-exposure prophylaxis if risk } \\
\text { factor; post-exposure prophylaxis } \\
\text { for all adults }\end{array}$ \\
\hline
\end{tabular}

$\mathrm{Td} / \mathrm{Tdap}=$ tetanus, diphtheria, acellular pertussis, MMR = measles, mumps, rubella, PPSV23 = pneumococcal 23-valent polysaccharide vaccine,

MPSV4 = tetravalent meningococcal conjugate vaccine 